\title{
Recurrent Subungual Viral Warts
}

\author{
Song Hyun Han (D), Soon Heum Kim (D), Dong In Jo (D) \\ Department of Plastic and Reconstructive Surgery, Konkuk University Chungju Hospital, Konkuk University School of Medicine, Chungju, Korea
}

\begin{abstract}
Warts, cutaneous manifestations of human papillomavirus infection, are difficult to cure. Warts have a high recurrence rate and there is no known single completely effective treatment. In this case, a 14-year-old boy presented with a subungual wart of the thumb that did not respond to salicylic acid. Excision was performed due to discomfort caused by pain and deformation of the nail plate. After the first operation, the patient had to receive two revision operations due to recurrence. Epithelialization was completed 2 weeks after the second revision surgery, after which there was no recurrence. According to literature review, many immunocompetent patients can expect spontaneous recovery without any treatment. If treatment is necessary, salicylic acid and cryotherapy have the strongest evidence of efficacy and are recommended as first-line treatments. Various other treatments are used alone or in combination. During surgical removal, special attention should be given for complete removal of the wart to prevent recurrence.
\end{abstract}

Keywords: Warts; Nails; Hand; Recurrent

\section{Introduction}

A wart is a cutaneous manifestation of a human papillomavirus (HPV) infection. Benign epithelial hyperplasia is caused by this virus, and a hard mass with hyper-keratinized appearance occurs. Trauma causes viral inoculation and localized infection. Warts commonly occur on the surface of the body, including the hand, foot, and elbow, and less commonly on the nail bed under the nail plate [1]. Subungual warts are an aggressive and destructive type of common warts. Because subungual warts are resistant to commonly used treatment modalities (topical salicylic acid application, cryotherapy, and laser cauterization), they are difficult to treat and cause damage to the nail matrix and may lead to nail deformity [2]. We report a case of a subungual wart recurring after excision and provide a literature review for the treatment modalities. The study was approved by the Institutional Review Board of Konkuk University Chungju Hospital (IRB No. KUCH 2020-07-021) and performed in accordance with the principles of the Declaration of Helsinki. Written informed consent was obtained from the patient for the publication of any data/associated images.

\section{Case}

A 14-year-old boy presented with a wart and pain of the right thumb. Before visiting our hospital, treatment with salicylic acid was performed, but there was no response. The wart invaded the sterile matrix of the nail bed from the hyponychium, and deformation of the nail plate was observed. The nail plate had exophytic growth and its surface was rough and hyperkeratotic (Fig. 1). The patient had no medical or trauma history.

\section{Case Report}

Received: July 14, 2020

Revised: September 9, 2020

Accepted: September 10, 2020

\section{Corresponding author:}

Dong In Jo, M.D., Ph.D.

Department of Plastic and Reconstructive Surgery, Konkuk University Chungju Hospital, Konkuk University School of Medicine 82 Gugwon-daero, Chungju 27376, Korea

Tel: +82-43-840-8860

Fax: +82-43-840-9862

E-mail: cozarmd@kku.ac.kr

This is an Open Access article distributed under the terms of the Creative Commons Attribution Non-Commercial License (https://creativecommons.org/licenses/by-nc/4.0/) which permits unrestricted non-commercial use, distribution, and reproduction in any medium, provided the original work is properly cited.

C) 2020 Korean Wound Management Society 

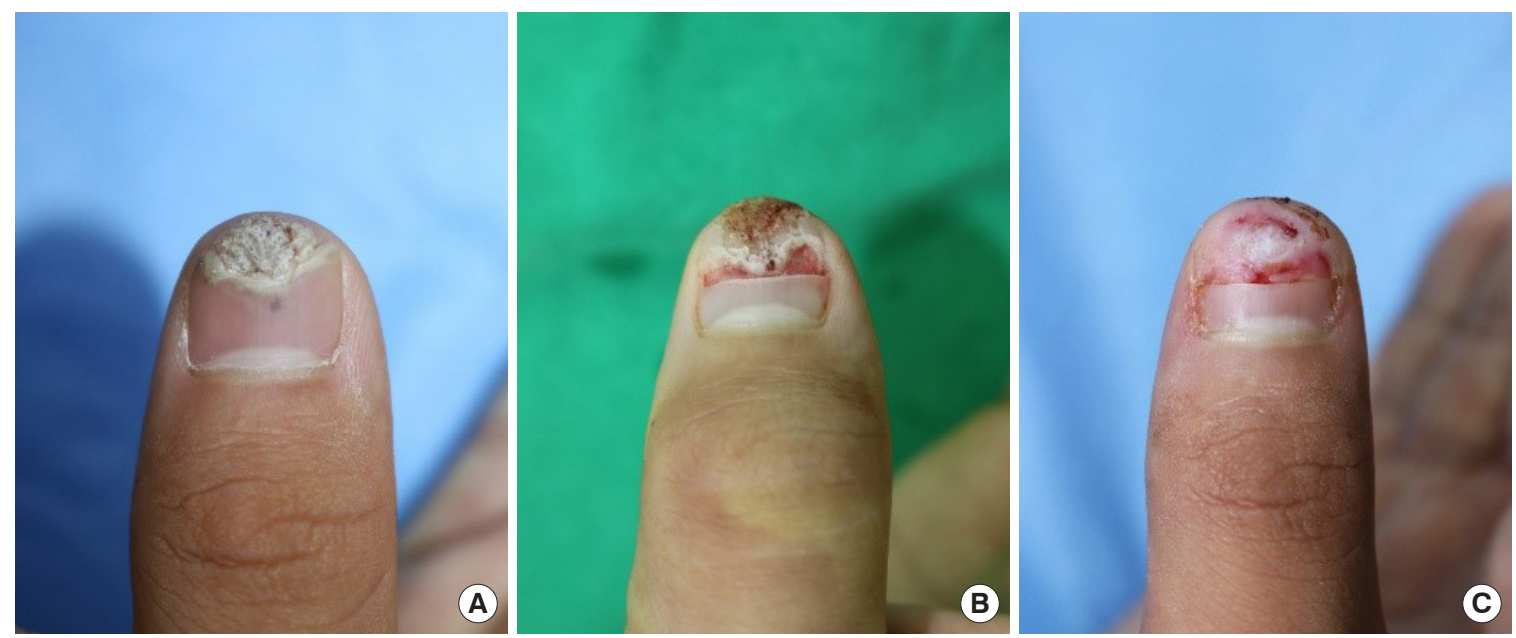

Fig. 1. Photographs before and after first procedure. (A) Hyper-keratinized wart with rough surface invades the sterile matrix of the nailbed. Deformation of the nail plate is also observed. (B) Intraoperative photograph after partial removal of nail plate. (C) Epithelialization in progress 6 days after surgery.
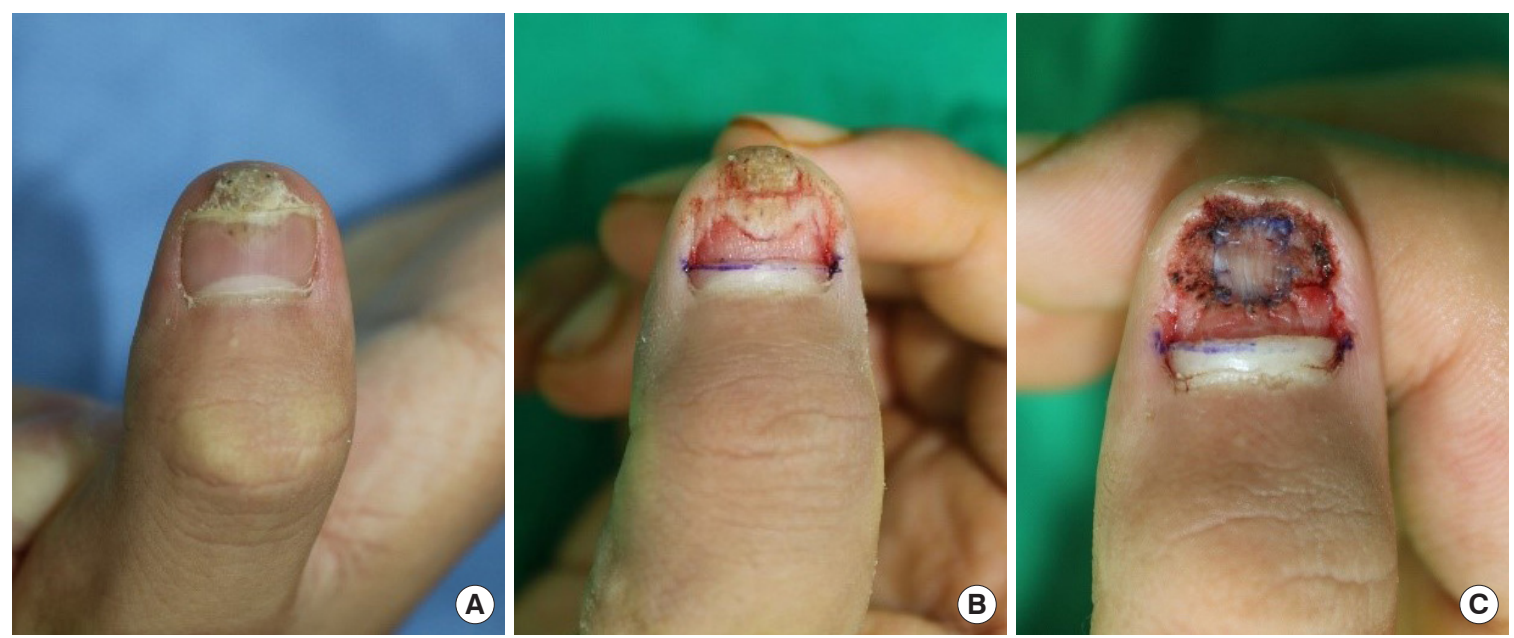

Fig. 2. First recurrent wart and photograph after revision operation. (A) Warts recurring on the same site. (B) Intraoperative photograph after partly removing the nail plate. (C) Immediate postoperative photograph after excision and nail bed graft.

Primarily, excision and curettage were performed using a number 15 blade and electrocauterization under a digital block. After surgery, epithelialization progressed with conservative treatment (Fig. 1). On the 20th day after surgery, recurrence was observed in the same area. Revision surgery was performed; excision and curettage using number 15 blades and electrocautery. A nail bed graft was additionally administered using the ipsilateral great toenail bed as the donor site (Fig. 2). A minor disruption was observed, but the epithelialization was complete. Two months after revision surgery, recurrent papillary growth was observed on the radial side of the hyponychium of the right thumb. After partial removal of the nail plate, the recurrent wart was observed on both sides of the sterile matrix of the nail bed. Excision and curettage were carefully performed through the reticular dermis to the superficial fascia using a number 15 blade and electrocauterization. Epithelialization was completed 2 weeks after surgery, and no recurrence was observed by 6 months follow-up (Fig. 3).

\section{Discussion}

Cutaneous warts are a common disease with high recurrence rates, and are difficult to treat, with no known treatment that is $100 \%$ effective. Various methods such as topical salicylic acid application, cryotherapy, pulsed dye laser therapy, surgical excision, and intralesional immunotherapy are used for its treat- 

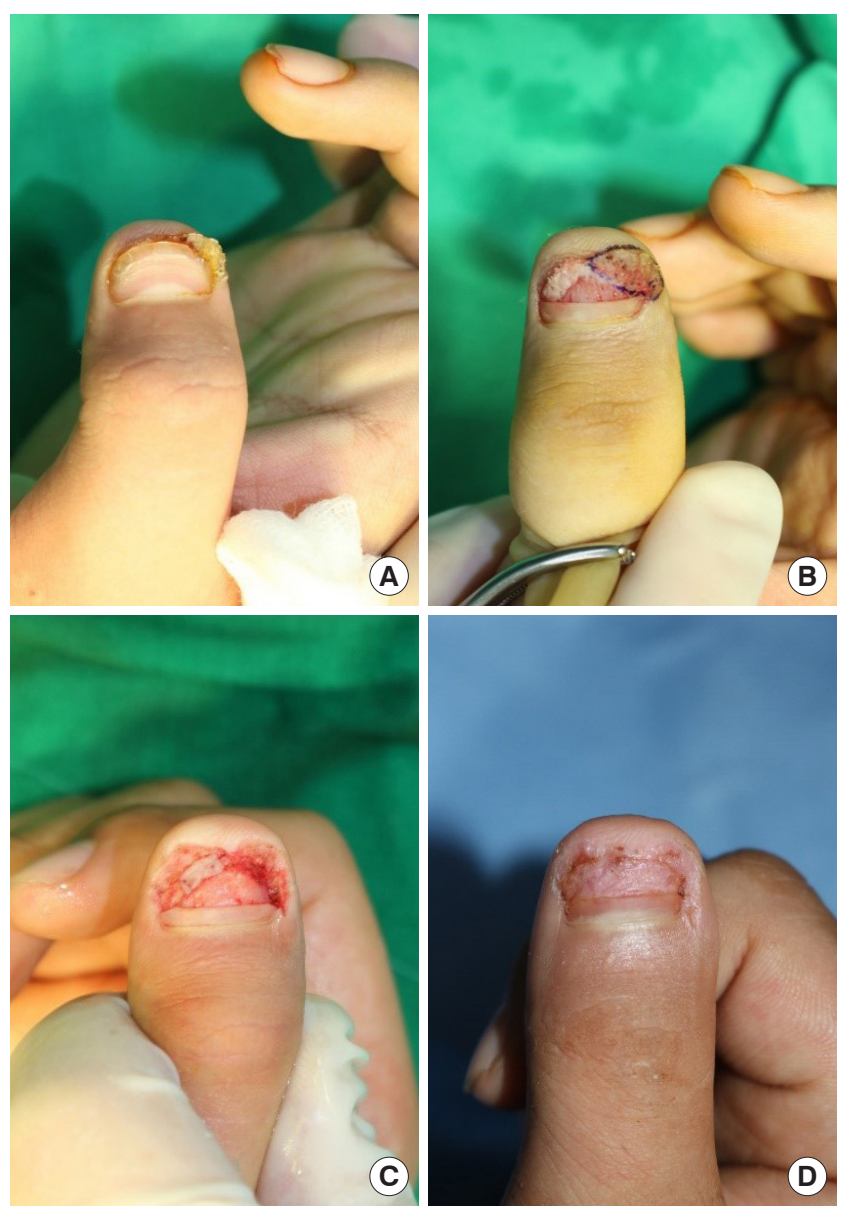

Fig. 3. Second recurrent wart and photograph after the second revision operation. (A) Relapsed warts on the ulnar side of the hyponychium. (B) Intraoperative photograph after partially removing the nail plate. (C) Immediate postoperative photograph after excision and curettage. (D) Epithelialization was completed 2 weeks after surgery.

ment. Depending on the symptoms and the site and size of the wart, it can be a minor problem, and in some immunocompetent patients the warts resolve spontaneously without treatment [3]. The cure rate within 10 weeks ranged from $20 \%$ to $30 \%$ in several studies. In observational studies, approximately half of the warts were spontaneously cured in 1 year and about two-thirds were cured in 2 years [4]. However, treatment is required when the patient experiences uncomfortable symptoms and cosmetic or functional problems. Among wart treatments, topical salicylic acid application has been most widely studied. It is the most effective method for wart treatment and is administered without a prescription. A meta-analysis of five studies conducted in 2011 reported that treatment with salicylic acid is 1.6 times more effective than that with a placebo [5]. According to a combined analysis of 16 studies, treatment

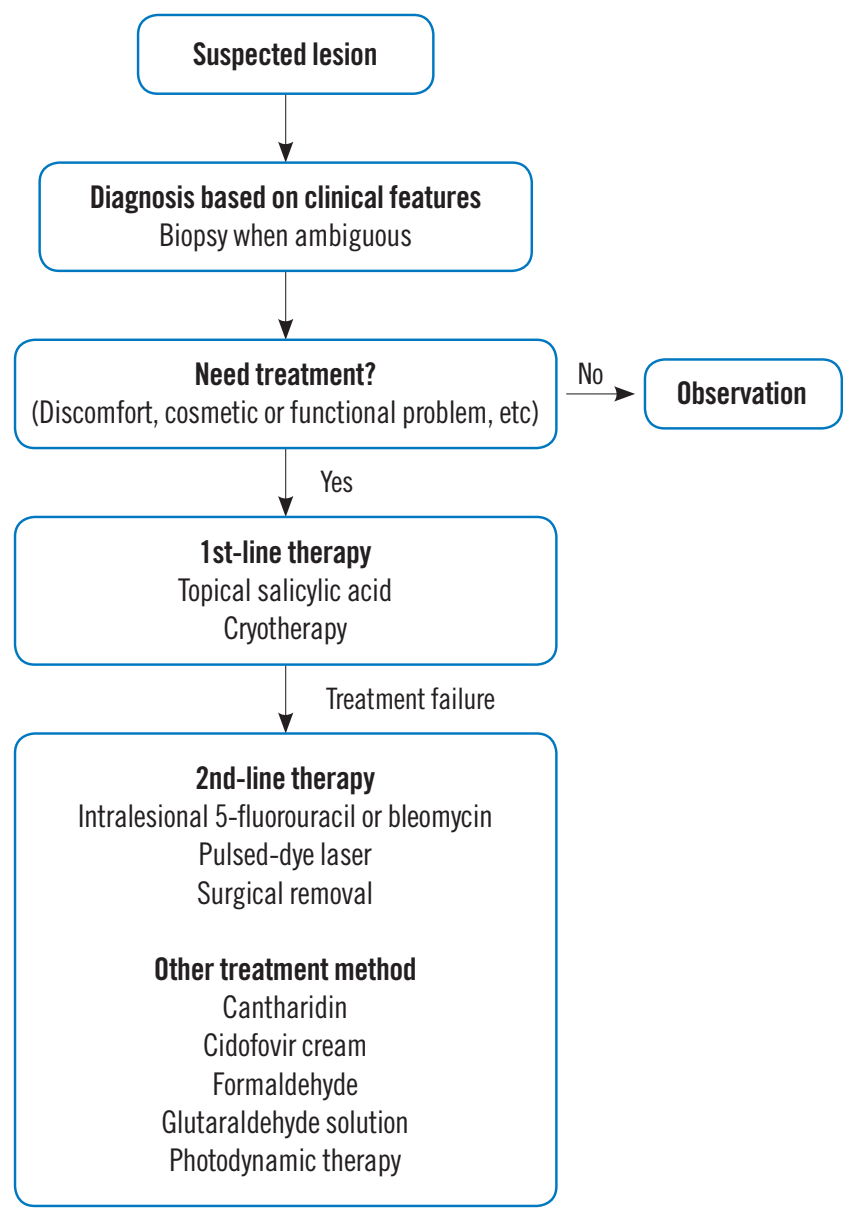

Fig. 4. Algorithm of our proposed hand wart management. The treatment modality is decided by considering various factors (number of wart, site, morphology, age, immune status, preference, etc). Destructive methods require sufficient hemostasis and proper wound management.

with salicylic acid had a mean cure rate of $49 \%$ from a range of $0 \%-69 \%$, while the placebo had a mean cure rate of $23 \%$ from a range of $5 \%-73 \%$. Therefore, salicylic acid is more effective than a placebo [5].

Meanwhile, the reported cure rate of cryotherapy in all sites ranged from $0 \%-69 \%$ and the mean was $49 \%$. According to a subgroup analysis, cryotherapy is more effective for hand warts than for plantar warts. In most studies comparing salicylic acid and cryotherapy, there was no significant difference in effectiveness. Aggressive cryotherapy causes more pain and blisters, but is more effective $[3,4]$.

Topical salicylic acid application and cryotherapy are recommended as first-line treatments because of their strong evidence of efficacy, while various other treatments are being studied. Chemotherapeutic drugs such as 5-fluorouracil and 
bleomycin can be used intralesionally, and some studies including a double-blinded randomized controlled trial report that their use shows higher cure rates than those using placebo [3]. Therefore, intralesional injection of 5-fluorouracil or bleomycin may be an effective treatment.

Another treatment in use, pulsed dye laser, can selectively destroy the wart vessels and has an evidence level of efficacy similar to that of the intralesional chemotherapeutic drugs. Some studies on the pulsed dye laser for wart treatment stated no significant difference compared with treatment with placebo or cryotherapy, while others show high cure rates $[3,4]$.

Although surgical removal (curettage or cautery) is also used, there is no high-quality published randomized controlled study. Success rates are reported as $65 \%-85 \%$ and scarring and recurrence rates are reported up to $30 \%$ after surgical removal. If the wart is removed by a destructive method, a raw surface appears and wound management is required. Also, as bleeding can be severe for surgical removals, it is recommended to complete the surgery with sufficient hemostasis. While there are not any established guidelines for wound management, Mahrle and Alexander [6] recommended light gauze dressing because occlusive dressing can worsen maceration and make the area susceptible to HPV infection. In addition, daily short bathing of the wound was also recommended to reduce secretion and prevent crust formation. According to studies with better results using photodynamic therapy or topical $1 \%$ cidofovir gel after removing the wart, a combination of different methods is recommended [7,8]. If sufficient hemostasis and proper wound management are performed during surgical treatment, surgical removal can be a good treatment option as it can immediately remove the entire wart.

There are no proven antiviral agents to treat warts [9]. However, there have been reports of cases where cutaneous warts disappeared after taking oral antiviral drugs (Acyclovir and Valacyclovir), and there also have been studies showing topical application to be effective, but the evidence is still weak $[3,10,11]$. Several other methods (Cantharidin, Cidofovir cream, Formaldehyde and Glutaraldehyde solution, Photodynamic therapy, etc.) have been studied, but there is still insufficient evidence for their effectiveness [4].

The location and shape of the wart indicate different types of HPV. HPV types 1, 2, 4, 27, and 57 cause the common wart (Verruca vulgaris) typically in the hands and feet $[3,12]$. The anorectal wart (Condyloma accuminatum) is mainly caused by HPV types 6 and 11, and the plantar wart (Verruca plantaris) on the soles is caused by HPV types 1, 2, 4, and 57 [13].
The cure rate of plantar warts is low because of the thickness of the stratum corneum, which reduces effectiveness of treatment in the sole [3]. Smooth and flat-topped plane warts smaller than $4 \mathrm{~mm}$ in diameter (Verruca plana) are caused by HPV types 3 and 10 [13]. Although the treatment of warts is not determined by the HPV type, the treatment method should be applied considering various factors including the number of warts, the site, and morphology, etc. $[12,13]$. For example, wart paint should not be used for facial warts because of severe irritation and the risk of scarring, and destructive methods can worsen plane (flat) warts because the plane wart is highly prone to spreading [13]. The algorithm for our proposed management of hand warts is presented in Fig. 4.

It is established that warts are caused by an HPV infection of keratinocytes in the outer layer of the skin, and HPV spreads through direct contact [3]. However, it is also claimed that the virus can spread through the blood as the HPV genome is detected in human peripheral blood mononuclear cells [14]. Recently, animal experiments have suggested the possibility of hematogenous spread of HPV [15]. Whether or not hematogenous infection is possible, bleeding during surgery can increase the chance of contact with HPV. Therefore, it is recommended to be careful not to cause bleeding when removing the wart (e.g., by using a finger tourniquet, or by pinching around the mass to reduce blood supply, etc.).

The topical treatment of subungual warts is difficult and can result in nail dystrophy; therefore, surgical removal is usually indicated [1]. In this study, topical salicylic acid application was performed, only to turn out ineffective. As the size of the wart increased the shape of the nail plate was steadily deformed. In addition, since the patient complained of pain, and because subungual warts can often develop malignant growths such as squamous cell carcinoma in situ, differential diagnosis was necessary, and therefore it was decided to perform surgical excision [1]. Despite the care taken during the first surgery and the two subsequent revision operations to completely remove the wart, relapses were observed after the initial surgery and first revision operation. However, no further recurrence was noted after epithelialization was complete after the second revision surgery. Treatment failure rates are reported to be up to $25 \%$ for simple warts and $50 \%$ for mosaic warts. Therefore, the surgeon must be aware that the wart is spread by direct contact and tends to recur a lot. Special attention should be paid to reduce the chance of contact and to completely remove warts during surgery. 


\section{Conflict of interest}

No potential conflict of interest relevant to this article was reported.

\section{ORCID iDs}

Song Hyun Han

Soon Heum Kim

Dong In Jo

https://orcid.org/0000-0002-2726-2583

https://orcid.org/0000-0001-9773-4753

https://orcid.org/0000-0002-3075-4482

\section{References}

1. Kline A. Subungual benign squamous papilloma: a case report. Foot Ankle J 2009;2:3.

2. AlGhamdi KM, Khurram H. Successful treatment of periungual warts with diluted bleomycin using translesional multipuncture technique: a pilot prospective study. Dermatol Surg 2011;37:486-92.

3. Sterling JC, Gibbs S, Haque Hussain SS, et al. British Association of Dermatologists' guidelines for the management of cutaneous warts 2014. Br J Dermatol 2014;171:696-712.

4. Mulhem E, Pinelis S. Treatment of nongenital cutaneous warts. Am Fam Physician 2011;84:288-93.

5. Kwok CS, Holland R, Gibbs S. Efficacy of topical treatments for cutaneous warts: a meta-analysis and pooled analysis of randomized controlled trials. Br J Dermatol 2011;165:233-46.
6. Mahrle G, Alexander W. Surgical treatment of recalcitrant warts. J Dermatol Surg Oncol 1983;9:445-50.

7. Wu L, Chen W, Su J, et al. Efficacy of the combination of superficial shaving with photodynamic therapy for recalcitrant periungual warts. Photodiagnosis Photodyn Ther 2019;27:340-4.

8. Orlando G, Fasolo MM, Beretta R, et al. Combined surgery and cidofovir is an effective treatment for genital warts in HIV-infected patients. AIDS 2002;16:447-50.

9. Fradet-Turcotte A, Archambault J. Recent advances in the search for antiviral agents against human papillomaviruses. Antivir Ther 2007;12:431-51.

10. Bagwell A, Loy A, McFarland MS, et al. Oral acyclovir in the treatment of verruca. J Drugs Dermatol 2016;15:237-8.

11. Tandeter H, Tandeter ER. Treatment of plantar warts with oral valacyclovir. Am J Med 2005;118:689-90.

12. Lipke MM. An armamentarium of wart treatments. Clin Med Res 2006;4:273-93.

13. Sterling JC, Handfield-Jones S, Hudson PM, et al. Guidelines for the management of cutaneous warts. Br J Dermatol 2001;144:4-11.

14. Bodaghi S, Wood LV, Roby G, et al. Could human papillomaviruses be spread through blood? J Clin Microbiol 2005; 43:5428-34.

15. Cladel NM, Jiang P, Li JJ, et al. Papillomavirus can be transmitted through the blood and produce infections in blood recipients: evidence from two animal models. Emerg Microbes Infect 2019;8:1108-21. 